\title{
Outcomes in partial sternotomy for aortic root surgery: the new routine procedure?
}

\author{
Amer Harky ${ }^{1}$, Runzhi Chen ${ }^{2}$, and Ahmed Othman ${ }^{1}$ \\ ${ }^{1}$ Liverpool Heart and Chest Hospital NHS Foundation Trust \\ ${ }^{2}$ Imperial College London
}

May 28, 2020

Outcomes in partial sternotomy for aortic root surgery: the new routine procedure?

Running Head: Partial sternotomy for aortic root repair

\section{Amer Harky ${ }^{1,2}$ MRCS, Runzhi Chen ${ }^{3}$ BSc, Ahmed Othman FRCS-CTh}

1. University of Liverpool, Liverpool, UK

2. Department of Cardiothoracic Surgery, Liverpool Heart and Chest, Liverpool, UK

3. Faculty of Medicine, Imperial College London, London, UK

\section{Corresponding author}

Amer Harky MRCS, MSc

Department of Cardiothoracic Surgery

Liverpool Heart and Chest

Liverpool, UK

E-mail: aaharky@gmail.com

Tel: +44-151-600-1616

Funding : none obtained

Conflict of interest : none declared

Key words : minimally invasive surgery, partial sternotomy, aortic root

Dear Editor,

We read with great interest the article by Elghannam et al.[1] in which they concluded that partial upper sternotomy (PUS) for aortic root surgery could be a safe alternative to full median sternotomy (FMS), albeit requiring longer operative times and greater operative skills.

Whilst we agree with some of the conclusions, there are aspects of this study that would benefit from further clarification. In our recent meta-analysis, we evaluated eight comparative studies for aortic root replacement with a total of 2,765 patients ( $\mathrm{n}=1974$ for PUS and $\mathrm{n}=1,791$ for FMS) [2]. PUS was associated with shorter cardiopulmonary bypass times, lower operative mortality, and shorter stays at intensive care and at hospital $(\mathrm{p}<0.05)$. However, no differences in aortic cross-clamp or total operation times were observed $(76.1 \pm 24.7$ versus $109.6 \pm 52.9$ minutes, WMD $-4.17,95 \%$ CI [-11.70, 3.37], $\mathrm{p}=0.28$ and $252.8 \pm 56.3$ versus $249.7 \pm 54.1$ minutes, $\mathrm{p}=0.31$ respectively). Similarly, no differences in re-exploration rates for bleeding were observed 
between PUS and FMS (OR 0.81, 95\% CI [0.55, 1.19], $\mathrm{p}=0.28$ ). It is also important to note that dialysis was more frequently required following FMS procedures ( $\mathrm{n}=2,217$ patients, $2.78 \%$ versus $3.36 \%, \mathrm{p}=0.001)$.

Thus, it would have been robust for the authors to include a patient-matched FMS group in order to facilitate direct comparison between the techniques. Moreover, the authors reported much higher mean cardiopulmonary bypass and cross-clamp times for PUS in comparison to results from our meta-analysis (101 \pm 33.5 versus $174 \pm 54.8$ minutes and $76.1 \pm 24.7$ versus $133 \pm 33.1$ minutes, respectively) [2]. Prolonged operative parameters are established risk factors for adverse perioperative outcomes [3]. Furthermore, it would be of interest to know if any patients required dialysis due to post-operative renal failure. This is important factor for quality of life and thus patient contentment, and useful for predicting long-term mortality.

Taken together, it would be pertinent to directly compare these results with an FMS control group to conclude whether PUS is indeed superior to FMS for aortic root surgery, an area which lacks robust evidence.

\section{References:}

1. Elghannam M, Aljabery Y, Naraghi H, Moustafine V, Bechte M, Strauch J, Haldenwang P. Minimally invasive aortic root surgery: Midterm results in a 2-year follow-up. J Card Surg . 2020;1-8. doi: https://doi.org/10.1111/jocs.14628

2. Harky A, Al-Adhami A, Chan JSK, Wong C, Bashir M. Minimally Invasive Versus Conventional Aortic Root Replacement - A Systematic Review and Meta-analysis. Heart Lung Circ. 2019;28(12):1841-1851. doi: https://doi.org/10.1016/j.hlc.2018.10.023

3. Harky A, Roberts N, Oo A. Minimal Access Techniques in Aortic Root Surgery. Int $J$ Cardiovasc Res . 2018;7(4). doi: 10.4172/2324-8602.1000357 